\title{
Balkanologie
}

Balkanologie Revue d'études pluridisciplinaires

Vol. I, n' ${ }^{\circ} 1$ | 1997

Volume I Numéro 1

\section{Nation, xénophobie et fantasme}

La logique de la violence nationale dans l'ancienne Yougoslavie

\section{Glenn Bowman}

Traducteur : Frédérique Humbert et Patrick Michels

\section{CpenEdition}

\section{Journals}

Édition électronique

URL : http://journals.openedition.org/balkanologie/201

DOI : 10.4000/balkanologie.201

ISSN : 1965-0582

\section{Éditeur}

Association française d'études sur les Balkans (Afebalk)

\section{Édition imprimée}

Date de publication : 1 juillet 1997

ISSN : 1279-7952

\section{Référence électronique}

Glenn Bowman, « Nation, xénophobie et fantasme », Balkanologie [En ligne], Vol. I, n 1 | 1997, mis en ligne le 24 novembre 2020, consulté le 17 décembre 2020. URL : http://journals.openedition.org/ balkanologie/201 ; DOI : https://doi.org/10.4000/balkanologie.201

Ce document a été généré automatiquement le 17 décembre 2020.

(c) Tous droits réservés 


\title{
Nation, xénophobie et fantasme
}

\author{
La logique de la violence nationale dans l'ancienne Yougoslavie
}

\author{
Glenn Bowman
}

Traduction : Frédérique Humbert et Patrick Michels

\section{NOTE DE L'ÉDITEUR}

Ce texte a été traduit de l'anglais par Frédérique Humbert et Patrick Michels avec l'aimable autorisation de l'auteur et l'aide de Paul Guyonnet et Mireille Robin. Ce texte est paru dans Goddard (Victoria A.), Llobera (Joseph R.), Shore (Cris), eds., The Anthropology of Europe. Identities and Boundaries in Conflict, Berg : Oxford, 1994, p.

143-171.

Les nationalismes exacerbés qui ont remplacé l'ethos communautaire yougoslave de bratstvo i jedinstvo ("fraternité et unité ») ont surpris les observateurs en 1991, lorsque la République Socialiste Fédérative de Yougoslavie s'est disloquée en ses Républiques constitutives. La sanglante guerre nationale qui s'en est suivie dans les territoires de l'ancienne Yougoslavie a substitué à l'enthousiasme avec lequel la plupart des Européens avaient salué l'effondrement de l'hégémonie communiste en Europe de l'Est un profond désarroi. En outre, le caractère des nationalismes de l'ancienne Yougoslavie constitue un défi face à l'optimisme avec lequel les théoriciens du nationalisme, à l'instar d'Eric Hobsbawm, annoncèrent la fin d'un phénomène dont ils croyaient - à la lumière du développement de l'économie globale - qu'il se réduirait à un atavisme. L'ouvrage élégiaque Nations et Nationalisme depuis 1780 d'Hobsbawm se termine sur une assertion qui, après Vukovar et Mostar, est symptomatique de l'orgueil tragique du modernisme :

L'histoire mondiale de la fin du XX ${ }^{\text {ème }}$ et du début du XXI ${ }^{\text {ème }}$ siècle (...) considérera les "États-nations" et les "nations" ou les groupes ethnolinguistiques essentiellement comme des forces en régression, résistant ou s'adaptant à la nouvelle restructuration supranationale du globe, ou bien étant absorbées ou disloquées par elle. (...) Le fait même que les historiens commencent enfin à progresser dans l'étude et l'analyse des nations et du nationalisme laisse entendre 
que, comme souvent, le phénomène a dépassé son zénith. La chouette de Minerve qui apporte la sagesse, disait Hegel, prend son vol au crépuscule. Qu'elle tournoie à présent autour des nations et du nationalisme est un bon signe. ${ }^{1}$

2 Considérés dans perspective globale, les nationalismes contemporains et les identités nationales qu'ils mettent en jeu semblent irrationnels, dans la mesure où les États nationaux qu'ils s'efforcent de mettre en place ne paraissent pas correspondre à la structure économique mondiale actuelle. Néanmoins, je soutiendrai qu'un tel point de vue est incapable de rendre compte de la force de séduction que peuvent avoir les rhétoriques nationalistes pour des personnes ayant à lutter au quotidien pour défendre et améliorer leurs conditions de vie. Dans les espaces domestiques où les gens existent et s'impliquent, les identités sélectives et les stratégies exclusives semblent être aussi puissantes et focalisées sur le but à atteindre qu'auparavant, sinon plus. J'analyserai dans cet article la logique de la haine nationale telle qu'elle se manifeste chez les nouvelles nations apparues sur le territoire de ce qui était la Yougoslavie afin de montrer que ce nationalisme ne peut pas être appréhendé dans les mêmes termes que le rationalisme moderniste décrit par les analystes. Je démontrerai plutôt qu'il est souvent inhérent aux discours politiques alliant passion et rationalité, si bien que le modernisme, qui nous offre de l'humanité une image sensée et rationnelle, est incapable de l'expliquer et d'en prendre la mesure. L'ancienne Yougoslavie peut constituer l'avant-garde d'une longue succession de conflits nationaux dans lesquels sombreront non seulement des territoires jusque récemment stabilisés par le régime communiste, mais aussi d'autres régions qui devaient leur stabilité politique à l'antagonisme global entre communisme et capitalisme. La compréhension des processus qui ont conduit au sanglant effondrement de la Fédération yougoslave permettra peut-être ainsi aux sociologues de concevoir de nouveaux modèles d'analyse de l'identité susceptibles de nous mener à comprendre la résurgence "irrationnelle" des communautarismes exacerbés et exclusifs, ainsi que les guerres intercommunautaires qu'ils entraînent.

\section{La quête du peuple}

3 La légitimité du système communiste fédéral a commencé à s'effondrer en 1988-1989, quand le dirigeant nationaliste serbe Slobodan Milošević a abrogé l'autonomie du Kosovo et de la Voïvodine et déposé le gouvernement du Monténégro. S'est ainsi inaugurée, dans les Républiques composant la Yougoslavie, une quête de nouveaux modes de légitimation des structures dirigeantes déjà en place (les appareils d'État de ces Républiques sont demeurés opératoires durant la transition des statuts républicains vers des statuts nationaux). Le discours dans lequel ce nouveau mode de légitimation s'est inscrit fut, sans exception, démocratique: les Yougoslaves, sous l'emprise de l'extase pro-occidentale qui balaya l'Europe de l'est après la chute du mur de Berlin, acceptèrent la panacée occidentale: ils virent dans les élections démocratiques le remède à tous les maux leur ayant été infligés sous le communisme. Les élections se déroulèrent en avril 1990 dans les Républiques de Slovénie et de Croatie, et en décembre dans celles de Macédoine, de Bosnie-Herzégovine, du Monténégro et de Serbie. Les résultats furent fort divers: la Slovénie et la Croatie votèrent pour des coalitions anti-communistes de centre-droit; les élections macédoniennes donnaient naissance à un parlement sans majorité, et, par conséquent, à un gouvernement de coalition incluant la vieille nomenklatura du Parti communiste, des réformistes et des 
nationalistes ; la Bosnie-Herzégovine établit une coalition entre Croates, Musulmans et Serbes excluant les communistes ; enfin, le Monténégro et la Serbie réinstallèrent leurs dirigeants communistes.

Les politiciens portés au pouvoir par ces élections déclarèrent que les programmes qu'ils souhaitaient appliquer étaient ceux que "le peuple" désirait réellement. Étant donné que la population n'avait guère eu, auparavant, son mot à dire dans les affaires de l'État, il n'existait pas de "plates-formes populaires" établies que les candidats auraient pu s'approprier ${ }^{2}$; le sentiment le plus répandu parmi la population des diverses républiques était tout simplement que le système précédent avait échoué et provoqué une diminution substantielle du niveau de vie, en particulier dans la décennie précédente caractérisée par un développement économique inégal et une dette extérieure élevée. En fait, la volonté populaire n'exprimait qu'une profonde insatisfaction: les programmes les plus convaincants à développer étaient donc ceux qui promettaient de trouver, et d'abolir, les causes de l'échec du système. L'effondrement soudain de l'hégémonie socialiste en Yougoslavie et dans toute l'Europe de l'est, qui, en général, n'avait pas été provoqué par la faillite économique, ni par une résistance interne organisée, a permis l'émergence de fantasmes populaires, dans certains cas millénaires; la population avait l'impression qu'en empruntant la voie magique de la démocratie prônée par l'Occident, elle se trouverait immédiatement dans un monde nouveau et, de loin, meilleur. Par conséquent, les plates-formes politiques ne furent pas organisées autour de plans rigoureux et sérieux envisageant des changements structurels dans les domaines politique et économique, mais autour de déclarations prophétiques: si les partis se présentant l'emportaient, ils transformeraient l'État en mode d'expression de la véritable volonté du peuple et ils excluraient de la nation tous les agents et organisations ayant perverti cette volonté dans le passé. Les élections présupposaient toutes que la légitimité déléguée par "le peuple" représentait la finalité inhérente du processus historique, et on l'opposa à celle revendiquée par les dirigeants communistes, dont le but était de mettre en œuvre l'idéologie communiste d'“État populaire". Par conséquent, la question centrale, au premier plan de toutes ces élections, fut « qui est le (vrai) peuple?».

5 La réponse fournie par les politiciens élus, qui était évidemment celle que la majorité des électeurs souhaitait entendre, fut que le vrai peuple était constitué des membres du groupe national dominant de chaque république. En Slovénie, par exemple, la coalition victorieuse de centre-droit, le DEMOS, soutenait, dans un programme nationaliste, que les Slovènes étaient fondamentalement industrieux et productifs et qu'ils deviendraient aussi riches que les capitalistes de l'Autriche voisine, dont ils étaient les émules, s'ils pouvaient se débarrasser de l'influence des communistes et des autres groupes nationaux ("du sud") non-slovènes qui interféraient dans leurs affaires. La campagne proposait, en fait, "la Slovénie pour les Slovènes » et ce programme était bien plus attrayant pour l'électorat que la plate-forme de la coalition de la gauche réformiste qui exigeait aussi bien l'intégralité des droits civils pour tous ceux résidant en Slovénie, que des changements radicaux, et pénibles, dans l'organisation sociale et économique. À l'autre extrémité de l'éventail politique, l'argument porteur dans les élections de Serbie, remportées par le parti national socialiste de Milošević, fut que les Serbes étaient de vrais communistes qui sauraient instituer une "Grande Serbie" aussi riche et puissante que l'avait été celle qui (dans les imaginations) avait régné sur une vaste partie des Balkans avant la conquête ottomane, s'ils n'en étaient pas empêchés 
par les conspirateurs étrangers anti-serbes (tel que le "croate" Tito que Milošević accusa d'avoir orchestré une conspiration "Vatican-Komintern" contre la Serbie). Le programme de la Ligue des forces de réforme conduite par Ante Marković, qui avait appliqué, en tant que Premier ministre fédéral, des réformes économiques radicales en 1989 et 1990, fut le seul programme "yougoslave" proposé à toutes les élections républicaines. La plate-forme politique de Marković appelait à " une Yougoslavie unie avec une économie de marché, le pluralisme politique, les droits et libertés démocratiques pour tous les citoyens $»^{3}$. Il fut écrasé dans toutes les élections, remportant seulement Tuzla, une ville industrielle de Bosnie-Herzégovine.

Deux éléments spécifiques jouèrent un rôle au sein des discours politiques des partis victorieux, communistes ou non. Le premier fut l'évocation de l'essence et de la volonté du "peuple" qu'on invoquait; le second fut la dénonciation de "l'autre", accusé d'empêcher le peuple de se réaliser pleinement et de le spolier des bénéfices susceptibles de découler de cette réalisation. Chacun de ces éléments faisait appel à une définition nationaliste de l'identité, élaborée en termes nationaux. Les élections yougoslaves furent remportées par les partis qui faisaient référence au peuple en termes d'identité nationale et rendaient responsables de ses problèmes des personnes et des groupes qui étaient, auparavant, des voisins (voisins, non seulement au sens où ils habitaient des républiques contiguës, mais encore, dans la plupart des cas, au sens littéral de voisins dans des communautés nationalement mixtes). Par ce procédé, on dressait des barrières entre des personnes qui avaient jusqu'alors vécu ensembles ou à proximité les unes des autres ${ }^{4}$. Ainsi le "nettoyage ethnique" fut promu en tant que programme politique dès les élections de 1990, dans la mesure où les esquisses théoriques des programmes politiques des partis victorieux ont été postérieurement mises en pratique en Croatie, en Bosnie-Herzégovine, et dans plusieurs régions de Serbie telles que le Sandžak ou le Kosovo. Cependant, il est important de souligner que ce projet fut orchestré par les élites politiques. Le fait de générer ces antagonismes nationaux fournit, d'une part, aux détenteurs du pouvoir un moyen facile de s'y accrocher et, d'autre part, aux aspirants au pouvoir la possibilité de parvenir à leur fin, ceci au moment où les moyens qui avaient servi jusqu'alors à prendre et à conserver le pouvoir étaient devenus caduques.

7 Toutefois, il ne suffit pas d'affirmer que ce projet fut "imposé" aux individus par des dirigeants immoraux et opportunistes. Il nous faut étudier l'enthousiasme avec lequel certains éléments de la population yougoslave acceptèrent de répondre à l'“appel" en se comportant en tant que nationalistes (ethnicistes) décidés à anéantir leurs voisins afin d'affirmer leur identité propre. Les brutalités qui ont caractérisé l'interaction nationale au cours des trois dernières années n'auraient pu, selon moi, être prévues par un observateur des modèles de coexistence qui ont caractérisé les années d'aprèsguerre. Après cette éruption de ferveur nationaliste, les mariages mixtes, la cohabitation et la coopération économique ont été remplacés par les mutilations (énucléation, arrachement des parties génitales), le viol des femmes et des enfants, le massacre de groupes nationaux dans les villes et les villages, la profanation et la destruction des biens et des maisons dont les propriétaires étaient perçus par les auteurs de tels actes comme appartenant à une "autre" nation, la réunion d'hommes, de femmes et d'enfants dans des camps de concentration où la torture, le meurtre et les privations génocidaires de nourriture et d'eau étaient ordinaires. De telles actions furent exécutées par des Serbes, des Croates et des Bosniens. Bien que la Slovénie n'ait pas connu de guerre nationale en raison de l'homogénéité relative de sa population, j'ai 
pu observer le harcèlement brutal par la police slovène de personnes non-slovènes, dont le seul crime était d'être sur le territoire de cette République. La haine nationale qui a explosée dans l'ensemble de l'ancienne Yougoslavie peut avoir été provoquée d'en-haut, mais la réponse populaire à cette fomentation fut enthousiaste. Tomaž Mastnak, philosophe slovène, indique que les milices de volontaires, ayant exécuté la plus grande partie des atrocités, ne sont pas des anomalies anti-sociales mais précisément des expressions du genre de société qui s'était développée dans l'ancienne Yougoslavie : « les milices représentent exactement la population en armes - la société civile dans son aspect le plus incivique $»^{6}$. L'analyse de la situation doit non seulement expliquer pourquoi les dissensions nationales ont permis si efficacement de saisir le pouvoir mais encore déterminer les raisons pour lesquelles l'appel aux armes contre les voisins se vit donner une réponse si passionnée.

\section{L'affirmation politique de la nation}

8 Lorsque de nouveaux États se séparent d'un État au sein duquel leur population avait été consolidée, déterminer quelle population appartient à quelle nation devient problématique. Dans le cas des nouveaux États ayant surgi sur le territoire de ce qui était la Yougoslavie, des frontières nettes entre "l'intérieur" et "l'extérieur" sont territorialement très mal définies. Les processus de modernisation qui ont bouleversé la Yougoslavie et sa population au XX⿳亠丷⿵冂丶 ${ }^{2}$ siècle n'ont fait qu'accentuer la mixité de populations que la coexistence sous les Empires ottoman et austro-hongrois avait déjà entremêlées ${ }^{7}$. La Bosnie-Herzégovine, peuplée de $40 \%$ de Musulmans, $33 \%$ de Serbes, $18 \%$ de Croates et $9 \%$ d'“autres" (une catégorie de recensement désignant les autres groupes nationaux et ethniques ainsi que les personnes refusant de se définir en termes ethniques ou nationaux) n'est pas une anomalie démographique: la Croatie était peuplée à $75 \%$ de Croates, $12 \%$ de Serbes et $13 \%$ d'“autres"; la Serbie, en dehors de ses provinces prétendues autonomes de Kosovo ( $10 \%$ de Serbes et $90 \%$ d'Albanais) et de Voïvodine ( $56 \%$ de Serbes, $21 \%$ de Hongrois et $23 \%$ d'“autres"), est peuplée à $75 \%$ de Serbes, $20 \%$ d'Albanais, $2 \%$ de Croates et $13 \%$ d'“autres". Et même la Slovénie, qui se considère nationalement homogène, est à $90 \%$ slovène, $3 \%$ croate, $2 \%$ serbe et $5 \%$ "autre". Lorsque d'importantes populations ne partageant pas l'identité nationale du groupe hégémonique résident sur le territoire de l'État que ce groupe tente de créer, le processus de légitimation de l'État en termes nationaux soulève la question du devenir de ces habitants qui n'ont aucun droit national à intégrer la collectivité politique. Lorsque les limites entre "nous" et "eux" ne correspondent pas aux lignes défensives des frontières territoriales mais coupent des villes et des villages et, bien trop souvent, des familles, "l'entité nationale" désirée peut être présentée comme envahie et occupée par des "ennemis" devant, au moins, être désarmés par la privation des droits civiques ${ }^{9}$ et, au mieux, être neutralisés par l'exil ou l'extermination.

9 Ce projet discursif de transformation des voisins en ennemis s'oppose au discours étatique dominant des 47 dernières années de "nationalité yougoslave" qui avait naturalisé la coopération et la consanguinité. Les traditions constitutives des identités depuis la Seconde Guerre mondiale étaient destinées à effacer les antagonismes intercommunautaires et établir le bratstvo $i$ jedinstvo yougoslaves comme le seul moyen viable d'assurer la survie et le bien-être des personnes. La Fédération yougoslave avait été établie sur le tracé de différentes frontières entre "l'intérieur" et "l'extérieur". La 
guerre des Partisans contre l'occupation nazie avait forgé une solidarité entre des individus de toutes les nations du pays pour la défense de la "patrie" et avait simultanément amené le parti communiste et son chef Tito au pouvoir. Elle fut suivie d'une purge brutale contre les "ennemis intérieurs", provoquant la mort de dizaines de milliers de Yougoslaves "collaborateurs" et l'exil de bien plus. Ultérieurement, les Ustaše, Croates ayant mené, avec le soutien nazi, une guerre nationale contre tous les non-Croates durant l'occupation, furent définis comme des "nazis" dans la rhétorique étatique (à savoir des collaborateurs d'un pouvoir étranger) et non comme des Croates. "L'autorité communiste entraînait le contrôle idéologique de la représentation du passé, [et] ces événements horribles qui pourraient perturber la nouvelle coopération interethnique ne devaient pas être mentionnés, sauf dans des catégories collectives "les victimes du fascisme", d'une part, et "les occupants étrangers et les traîtres locaux", d'autre part. $»^{10}$

La scission entre la Yougoslavie et l'Union soviétique en 1948 fournit une autre impulsion à la solidarité yougoslave lorsque «la plus grande partie de la nation s'est ralliée à Tito face à la menace soviétique ${ }^{11}$. Les développements ultérieurs de la politique étatique maintinrent la Yougoslavie "en équilibre" entre l'est et l'ouest. Les intérêts de la nation, et des divers individus la constituant, pouvaient être ainsi toujours établis contre les conspirations d'une multitude d'ennemis menaçant la Yougoslavie en dehors de ses frontières territoriales et idéologiques. Ainsi, les discours à propos d'antagonismes nationaux ne pouvaient pas aisément mobiliser une longue tradition d'hégémonie pour justifier la division en communautés dans la mesure où la tradition de l'État hégémonique communiste soutenait que la survie des nations yougoslaves dépendait au contraire de leur coopération à sa défense. Les agents désirant établir des identités exclusivement nationales devaient donc "inventer" des traditions de haines irréversibles pouvant justifier des redéfinitions radicales dans les domaines de la sociabilité et de la coopération ${ }^{12}$. Cependant, une telle invention n'impliquait pas l'invocation de motifs de haine ex nihilo. Les constitutions successives (il y en eut quatre, la dernière datant de 1974) ont maintenu les marqueurs d'identité nationale dans la fédération, et nombre d'incidents et d'événements de l'histoire récente, et plus ancienne, de la Yougoslavie pouvaient être rappelés et interprétés de manière à fournir une base aux arguments signifiant que les voisins putatifs étaient en fait, à cause de leur identité nationale différente, des ennemis de sang déguisés. Il ne s'agissait donc pas tant de l'“invention" de traditions d'antagonismes intercommunautaires que d'une modification discursive permettant aux "mémoires" périphériques et assourdies de devenir les points centraux de nouvelles définitions identitaires. Démographiquement la Yougoslavie était composée de six groupes nationaux dominants (Slovènes, Croates, Serbes, Monténégrins, Macédoniens et Musulmans) et de douze minorités nationales (Albanais, Hongrois, Turcs, Slovaques, Tsiganes, Bulgares, Roumains, Ruthènes, Tchèques, Italiens, Valaques et Ukrainiens) dispersés sur un territoire caractérisé par des histoires régionales diverses et des variations considérables de niveau de richesse. Sous Tito, six républiques étaient reconnues : cinq correspondant à la prédominance d'un groupe national et une (BosnieHerzégovine) peuplée de trois principales communautés nationales (Croates, Serbes et Musulmanes). En outre, deux régions autonomes (Kosovo et Voïvodine) avaient été créées en reconnaissance de la population majoritaire albanaise au Kosovo et de la grande proportion de Hongrois en Voïvodine. Les nations dominantes peuvent, pour la plupart, être différenciées en termes de religion et/ou de langue : ainsi les Slovènes 
sont catholiques et parlent le slovène, les Croates sont catholiques et locuteurs du serbo-croate (la langue "croate" se distingue essentiellement du "serbe" par son alphabet latin au contraire du cyrillique de la langue "serbe"), les Serbes parlent serbocroate et sont membres de l'Église orthodoxe serbe. Non seulement les membres d'une communauté "nationale" peuvent vivre dans la république d'une autre "nation", mais on trouve aussi des anomalies catégorielles telles que des Serbes catholiques. De surcroît, certaines nations paraissent être plus le produit de la politique de l'État communiste que de distinctions culturelles "naturelles". Ainsi, par exemple, les Monténégrins sont reconnus en tant que communauté nationale alors qu'ils parlent le serbo-croate et partagent l'affiliation orthodoxe de leurs voisins serbes. Les Macédoniens, disposant d'une langue distincte, n'ont adopté une structure religieuse distincte de celle des Serbes et des Monténégrins qu'en 1967 à travers les machinations de l'État yougoslave ${ }^{13}$. Les Musulmans, "nation" sans base territoriale parlant serbocroate, ne se virent donnés un statut national qu'en 1968 afin « de les soustraire à la compétition entre Serbes et Croates destinée à démontrer que leur identité "réelle" était soit serbe soit croate (...) [et de] neutraliser leurs aspirations territoriales sur la Bosnie $»^{14}$.

11 Ainsi, comme le révèle l'anomalie "nationale" des Musulmans, formant un groupe national sans territoire national, l'offre du statut national était un stratagème discursif servant, dans certains cas (comme lorsque les Serbes et les Croates revendiquaient nationalement la Bosnie-Herzégovine en affirmant que les Musulmans sont des Serbes ou des Croates s'étant convertis à l'islam sous l'occupation ottomane ou lorsque les Serbes soutiennent que les Macédoniens ou les Monténégrins sont "réellement" des Serbes) à entraver les revendications nationales et, dans d'autres, à fournir une concession stratégique aux groupes nationaux consolidés dans un État multinational. Dans ce cas, l'État communiste fournissait une rhétorique dans laquelle la population pouvait déclarer une identité nationale n'ayant été ni complètement assimilée, ni dominée par cet État et qu'elle désirait affirmer. Le patronage étatique de ces nouvelles "identités", constituant un moyen de dispersion du potentiel de solidarités antiétatiques en dehors de la fédération, encouragea la soumission des identités nationales à l'identité englobante fournie par l'État yougoslave. Ce dernier entretenait de cette façon ces identités en tant qu'“étendards vides", comme les qualifie Edwin Ardener ${ }^{15}$. Ces signes d'identités n'étant pas reliés à des programmes spécifiques peuvent être mobilisés comme symboles et se voir donner des contenus appropriés lorsque des situations favorables surviennent. Ainsi, les identités nationales servirent, durant la période de l'État hégémonique, de moyens d'expression des conflits régionaux (surtout économiques) ne pouvant être exprimés dans la rhétorique de la fédération communiste unifiée ${ }^{16}$. Néanmoins, à la fin des années 1980, lorsque l'idéologie étatique perdit aussi bien sa légitimité que son pouvoir de contrôle des dissensions régionales et lorsque les problèmes du développement économique inégal des Républiques se tranformèrent en bases de l'expression de l'opposition à l'ordre ancien ${ }^{17}$, ces identités nationales fournirent les fondements discursifs sur lesquels purent s'appuyer une activité politique. Le mécontentement vis-à-vis du gouvernement central, provoqué par ce qui était perçu comme des injustices concernant tous les habitants d'une région, indépendamment de leur appartenance nationale, pouvait ainsi être plus aisément articulé en termes "nationaux". Cela indiquait avec certitude que ce serait la voie nationaliste, plutôt qu'une autre, qui serait choisie pour sortir de l'impasse de la politique communiste. Avec l'autodestruction effective du communisme, l'origine des 
désastres du passé et des privations du présent devait être recherchée dans le champ des antagonismes nationaux.

\section{Le discours national}

12 Le processus de redéfinition officielle des discours sur l'identité, ainsi que les implications politiques de ces transformations débuta dans les années 1980, alors que Milošević, pour assurer son ascension au pouvoir en Serbie, soulevait l'animosité populaire contre les Kosovars albanais en diffusant le sentiment que les "musulmans" menaçaient, comme au XIV ème siècle, d'expulser les Serbes de leur patrie historique du Kosovo. La presse officielle serbe commença à publier des histoires rapportant des cas de viols de femmes serbes par des "musulmans" albanais du Kosovo, de profanations de monastères orthodoxes, ainsi que de fréquentes allégations d'expulsions de familles serbes de leurs maisons et de leurs terres, autorisées par des fonctionnaires albanais forts du statut d'autonomie du Kosovo, de telle sorte que ces propriétés pouvaient être reprises par des immigrés illégaux de l'Albanie voisine ou par les enfants des Kosovars prolifiques ${ }^{18}$. Simultanément, il y avait une reconnaissance et une promotion officielles des vieilles traditions (qualifiées de "folkloriques" sous le régime de Tito) rappelant la lutte héroïque de la nation serbe contre l'invasion des armées ottomanes. Vidovdan, la célébration annuelle de la défaite des armées du Prince Lazar Hrebeljanović par les troupes ottomanes sur le "champ des merles" le 15 juin 1389, devint une cérémonie officielle au cours de la période qui conduisit à l'abrogation de l'autonomie kosovare. Les principaux membres du gouvernement serbe, dont Milošević, écoutaient les ménestrels villageois chantant le destin tragique des héros chrétiens ayant péri 600 ans plus tôt en défendant la Serbie contre l'invasion étrangère, avant de tenir des discours enflammés sur le thème de "plus jamais ça ». Lors du Vidovdan de 1989, alors que l'autonomie du Kosovo était abolie et qu'un état de siège effectif y était appliqué, les reliques du Prince Lazar, ayant reposé en Serbie depuis sa défaite six siècles auparavant, firent l'objet d'un défilé rituel à travers les villes et les monastères de Serbie avant de "retourner" en grande fanfare au monastère orthodoxe de Gračanica au cœur du Kosovo.

L'articulation du discours serbe, fondé sur l'opposition aux Albanais, servit à reconstituer la "Serbie" comme un lieu d'identité et les "intérêts serbes" comme un centre de préoccupation. Parallèlement aux événements en Serbie, la Croatie entrait elle aussi dans une phase nationaliste où la définition de la communauté et de ses intérêts occupaient une place centrale. D'une part pour répondre à la menace perçue du nationalisme serbe et d'autre part pour accéder au pouvoir, les politiciens nationalistes appelaient à la séparation de la Croatie de la fédération yougoslave en prétextant que, sous le communisme, la totalité des Croates avaient été punis pour les activités des Ustaš $e^{19}$ et qu'ils avaient, par conséquent, vu leurs droits en tant que Croates et Yougoslaves supprimés par l'État "dominé par les Serbes ». Afin d'étayer cette thèse, les nationalistes invoquaient la mémoire du "printemps croate" de 1971 (un mouvement significatif ayant exigé une plus grande décentralisation politique et une plus grande autonomie financière pour la république de Croatie). Ils soutenaient qu'aussi longtemps que le gouvernement central serait sous le contrôle des Serbes, ces derniers continueraient de nier aux Croates leurs droits historiques en tant que peuple. En 1989 Franjo Tudjman, ancien partisan communiste devenu président du nouveau 
parti «communauté démocratique croate » (HDZ - Hrvatska Demokratska Zajednica), et Président actuel de la Croatie, nettoya l'image nationale croate (souillée pendant des années par l'équation entre le fascisme ustaša et le nationalisme croate) en annonçant à Jasenovac (site du plus célèbre camp d'extermination ustaša) que les exactions ustaše n'avaient jamais été aussi étendues que la propagande d'État l'avait soutenu et, en outre, qu'elles n'étaient pas distinctes des autres brutalités commises à cette époque ${ }^{20}$. Par la suite, la presse, aussi bien en Croatie qu'en Slovénie, valida en apparence cette dernière affirmation en publiant les images des corps de milliers de victimes des massacres perpétrés par les Partisans après la fin de la guerre. Il s'agissait des collaborateurs croates et slovènes, des Serbes anti-partisans, Četnici (Tchetniks), ainsi que des membres de leurs familles respectives, ayant fui la Yougoslavie devant les forces partisanes victorieuses pour être finalement confiés à ces dernières par les troupes britanniques. Les photos de grottes pleines d'ossements empilés inondaient les journaux des deux Républiques donnant raison aux rhétoriques de la campagne électorale. En effet, ces morts, référés auparavant en termes non-nationaux tels que "nazis" ou "quislings", étaient devenus des "victimes croates" ou des "victimes slovènes" de la brutalité communiste.

Le programme nationaliste slovène était, à la base, simplement un programme anticommuniste, et le caractère positif de l'identité slovène restait à inventer. Dans la période précédant le vote pour l'indépendance, des symboles identitaires furent passés en revue, incluant, avec le plus grand succès, le kozolec, dispositif pour sécher le foin particulier à certaines régions de Slovénie. À la veille de la proclamation de l'indépendance, des discussions échauffées avaient encore lieu dans le Parlement à propos du drapeau que devrait adopter le nouveau pays. La plupart des modèles suggérés étaient refusé par le parlement parce que ses membres pouvaient y discerner des traces du drapeau de l'ancienne Yougoslavie. Dans le cas slovène, c'est l'absence de haines nationales spécifiques mobilisables envers un groupe voisin qui a permis à la Slovénie d'échapper à la guerre inter-communautaire ayant dévasté le reste de l'ancienne fédération. Cette absence a contribué à la chute de la droite nationaliste dans la période qui a suivi l'indépendance. L'un des points centraux du programme de la coalition élue DEMOS était l'opposition à l'avortement sous le prétexte que «la Slovénie est un petit pays entouré de grands ennemis, et [que] les femmes ne devaient pas avoir le droit d'avorter de futurs défenseurs de la nation ». Cependant, un nombre conséquent de femmes se sentirent moins menacées par un ennemi extérieur que par cette tentative d'abroger leur pouvoir sur leur corps. Cette nouvelle opposition engendra de nombreux groupes pro-avortement qui se joignirent à d'autres partis d'opposition dans une union qui finit par renverser la majorité parlementaire du DEMOS et par instaurer une coalition libérale en grande partie concernée par les problèmes domestiques ${ }^{21}$. Ainsi, tandis qu'en Slovénie la conduite de l'indépendance était alimentée par l'antipathie envers le communisme et la fédération imposés à la Slovénie, une fois le vieil ordre désintégré, les Slovènes se retrouvaient sans la distraction commode d'ennemis extérieurs et avec la tâche difficile de créer une identité nationale viable pour eux-mêmes.

En Croatie, au contraire, l'"étendard vide" que les partis anti-yougoslaves soulevèrent en opposition à l'État yougoslave se trouva bientôt recouvert des emblèmes des luttes collectives antérieures. Malgré le passé partisan de Tudjman et ses tentatives d'exorciser les fantômes des Ustaše du nationalisme croate, il adopta beaucoup d'éléments du programme et des symboles de l'État Indépendant de Croatie ustaša dès 
qu'il dut articuler un programme pour la HDZ. Lors de la campagne présidentielle du printemps 1990, Tudjman et la HDZ appelèrent à la formation d'une Croatie indépendante dans ses "frontières historiques" (donc incluant la plupart de la BosnieHerzégovine), ayant un drapeau national sur lequel l'étoile rouge de l'État yougoslave serait remplacée par le "damier" (šahovnica) qui avait orné le drapeau national de l'“État Indépendant de Croatie", et possédant une langue croate purifiée de tout mot "serbe". Selon Denich, il affirma également que l'« État Indépendant de Croatie de la Seconde Guerre mondiale n'était pas (...) une formation "quisling", mais une "expression des aspirations historiques du peuple (nation) croate" à posséder son propre État indépendant $\aleph^{22}$. La "positivité" de l'identité croate fut réalisée par l'adoption d'une identité croate anti-yougoslave ayant existé antérieurement, et cette affirmation de l'apparat de la "vraie" Croatie terrifia évidemment les Serbes vivant en Croatie. Devant eux, se concrétisait, de nouveau, le même ordre cauchemardesque sous lequel ils, ou leurs parents, avaient souffert entre 1941 et 1945.

Encore une fois, les ossements jouèrent un rôle réel dans la constitution de l'identité lorsque les Serbes de la région de Krajina en Croatie invitèrent des journalistes et des photographes locaux et de Serbie à se présenter devant les fosses où les squelettes des Serbes de Krajina massacrés par les Ustase avaient été ensevelis ${ }^{23}$. Non seulement ces monuments retraçant le destin des Serbes de Croatie sous les Ustaše servirent à légitimer la résistance locale des Serbes de Croatie au nouvel ordre croate (une résistance qui conduisit à l'auto-proclamation de la Krajina, par la force des bras et le "nettoyage ethnique", en tant qu'État serbe indépendant bien qu'internationalement non reconnu), mais ils fournirent aussi un point focal d'articulation à la haine nationale envers les Croates en Serbie. Denich indique que si

les rébellions des communautés serbes en Croatie étaient motivées par leurs propres mémoires du régime oustachi, maintenant étrangement réincarnées dans les déclarations et les symboles du nouveau gouvernement nationaliste (...), les habitants de la Serbie n'avaient pas éprouvé la terreur oustachie, et leurs souffrances de guerre provenaient des mains des Allemands et des autres occupants étrangers, plutôt que des Croates. En conséquence, il n'y avait que peu de réel sentiment anticroate en Serbie. ${ }^{24}$

Néanmoins, le régime de Milošević soutenait que les Serbes de Serbie reconnaitraient leur propre destin potentiel aux mains des "Croates" dans celui des Serbes de Croatie morts 45 ans plus tôt. Les médias serbes contrôlés par l'État présentèrent régulièrement, à la télévision et dans les journaux, des images des corps. Ainsi que je l'ai constaté lorsque j'étais à Belgrade aux premiers jours de la guerre, les maisons d'édition officielles emplissaient les librairies de livres en plusieurs tomes, abondamment illustrés, réexaminant l'histoire jusqu'alors interdite de la tentative "croate" d'exterminer les "Serbes". Les Serbes de la Serbie étroite, déjà convaincus par la campagne de haine orchestrée par le régime contre les Kosovars qu'ils étaient, en tant que Serbes, près de perdre leur patrie ancestrale (non pas, notons-le, leurs propres maisons, mais le foyer du peuple serbe), s'entendaient désormais dire qu'ils étaient, en tant que Serbes, près de perdre leurs vies ${ }^{25}$. Avec la promulgation fructueuse de la branche du national socialisme de Milošević, qui impliquait la mise en circulation de traditions auparavant discréditées et les histoires d'atrocité auparavant étouffées, les Serbes gagnaient la promesse d'une "Grande Serbie", que la menace de son vol rendait plus nécessaire, et la fraternité du "peuple serbe", que les images de son extermination sollicitaient. Comme ceux qui suivirent les sirènes du nationalisme dans d'autres 
régions de l'ancienne Yougoslavie, le peuple serbe se voyait promis un futur utopique en échange d'un engagement dans une longue lutte qui détruirait les ennemis de cet avenir.

Ce que Milošević, Tudjman et d'autres politiciens nationalistes ont gagné en jouant la carte nationale dans leur quête pour le pouvoir semble clair. En substituant au discours du champ social basé sur la cohabitation et la coopération (" unité et fraternité ») celui fondé sur l'exclusion et la guerre nationale («sang et territoire »), ils ont été capables, tout d'abord, d'amener l'intérêt du peuple sur un plan où il était défini en termes essentialistes, l'intérêt propre de chacun dépendant du fait d'être "Serbe", "Croate", "Slovène", etc. Lorsqu'une personne doit s'imaginer principalement comme un représentant d'une collectivité nationale, toute menace à cette collectivité (menace contre son pouvoir, la vie ou la propriété d'un de ses membres présenté comme tel) est simultanément une menace contre cette personne. Non seulement il ou elle perçoit le co-national menacé comme "le même que" lui/elle-même, mais il ou elle imagine aussi que son ennemi est également celui de tous ceux (il ou elle inclus) qui partagent l'identité du menacé ${ }^{26}$. L'ennemi n'attaque pas le peuple en tant que tel; il attaque les "Serbes", les "Croates", ou les "Slovènes".

Le deuxième avantage procuré par la carte nationale est que l'invocation d'un ennemi (ou d'une multitude d'ennemis) permet aux politiciens de faire porter la responsabilité de ces problèmes sur l'ennemi visible, tandis que les problèmes sociaux qui avaient provoqué le mécontentement initial envers le régime communiste sont demeurés en vigueur et ont, dans la plupart des cas, réellement empiré. Par conséquent, il n'est pas nécessaire de s'attaquer à la tâche difficile de restructurer la société en Croatie ou en Serbie; tout ce qu'il fallait faire, pour convaincre la majorité de la population qu'on avait réalisé des pas en avant, était de mener une guerre contre le ou les ennemis. On pourrait soutenir que c'est la guerre qui maintient les régimes nationalistes au pouvoir. Si la guerre devaient s'arrêter, il serait de plus en plus difficile d'attribuer l'appauvrissement radical et croissant des populations de Croatie et de Serbie aux actions de leurs ennemis, et la corruption et l'inefficacité des cliques dominantes deviendraient apparentes.

Le dernier avantage obtenu par la direction nationaliste en évoquant un monde structuré autour d'une opposition absolue, quasi "ontologique" 27 , entre "eux" et "nous" découle de ce manichéisme. Dans l'ancienne Yougoslavie, les dirigeants nationalistes affirment qu'il est nécessaire d'abroger les droits des personnes qu'ils dirigent, prétextant qu'un pouvoir absolu est nécessaire pour détruire l'ennemi absolu du peuple. Si l'ennemi est la source de tous les maux, et si le "nous" devant exister une fois ce mal éradiqué, est fondamentalement bon, alors la direction menant la lutte, au cours de ces sales journées, pour détruire ce mal est elle-même la personnification du principe du bien. L'élévation de la direction nationaliste, et particulièrement du "Chef" per se, au statut d'“agent de la rédemption" est évidente dans les brandissements passionnés d'affiches de Milošević pendant les manifestations nationalistes serbes et, de manière plus saillante, dans l'emplacement de statuettes de Tudjman à côté de celles de la Vierge Marie dans les boutiques de souvenirs au centre croate de pèlerinage, Medjugorje $^{28}$. Le dirigeant se présente comme la représentation charismatique de la "volonté du peuple" et, aussi longtemps qu'on croit qu'il représente cette volonté, toute activité qu'il initie sera perçue comme "nécessaire" pour son rachat. Une telle légitimation du pouvoir peut être sapée de deux manières. La première survient 
lorsque les personnes perdent leur foi dans l'existence du mal servant à justifier la violence et la répression de l'État, comme cela a été le cas en Slovénie. La seconde se produit lorsque les personnes perdent leur foi dans le dirigeant en tant que représentant charismatique du principe de la nation, et le défi lancé à Milošević par Vojislav Šešelj du Parti radical serbe d'extrême droite aux élections parlementaires du 19 décembre 1993 en Serbie est fondée sur une telle réévaluation. Ici, le dirigeant peut être présenté comme un "faux messie" et sa place peut être usurpée par une autre personne dont la brutalité marquée et l'extrémisme semblent plus à même d'exprimer la violence dont la nation a besoin pour détruire la force menaçant de l'anéantir.

21 Les discours des directions nationalistes au sujet de l'ennemi, largement diffusés par les médias qu'elles-mêmes contrôlent, créent, effectivement, un monde partagé entre deux camps où il n'existe aucun endroit neutre. Ainsi, quiconque ne soutient pas la direction nationale est nécessairement un partisan de l'ennemi (cette logique a justifié la répression extrême des antinationalistes en Serbie et en Croatie, ainsi que les meurtres brutaux commis par des Bosniaques serbes et croates de co-nationaux ayant refusé de prendre les armes pour soutenir la cause nationale) et tous les éléments du champ social doivent être interprétés en termes de camp auquel ils appartiennent. Une histoire très connue en Bosnie relate un échange de graffitis sur la frontière contestée entre les secteurs serbe et bosnien de Sarajevo. Quelqu'un écrivit sur un mur de la poste centrale, se dressant sur cette limite frontalière, « Ici, c'est la Serbie » et, bientôt, quelqu'un d'autre repeignît ce message, le remplaçant par «Ici, c'est la Bosnie ». Un troisième interlocuteur biffa le deuxième message et écrivit à sa place «Ici, c'est un bureau de poste! ». Les autres tentatives de revendication et de nettoyage de régions des signes souillants de la présence d'autres sont moins humoristiques; je ne fais pas, ici, seulement référence au "nettoyage ethnique" per se, mais aussi à son aménagement corollaire mettant en jeu des escouades de volontaires venus de Serbie sur des territoires de Bosnie ayant été pris et purgés des Musulmans par les milices serbes afin, non seulement, de détruire les mosquées, mais encore de labourer le sol sur lequel elles se trouvaient, d'y planter des arbres, et d'y construire des aires de jeux. En se fondant sur les démentis faits par la suite aux visiteurs quant à l'existence d'une mosquée en ce lieu, on peut interpréter pragmatiquement ce remodèlement du paysage comme une tentative pour masquer le "nettoyage ethnique" qui a eu lieu. Cependant, dans la mesure où les visiteurs sont supposés savoir que des Musulmans ont vécu ici, je soupçonne que la refonte du paysage sert à créer, pour les Serbes eux-mêmes, l'image d'un monde nouveau ne portant aucun signe de l'histoire de laquelle il est violemment né. Cette élision du processus historique est un élément nécessaire du discours de légitimation de la violence utilisée pour la création de communautés "nettoyées". L'institution de la "vraie" Serbie est un "retour" à un état de pureté ontologique et un tel état doit être dépourvu des marques de la condition profanée et "irréelle" dans laquelle la "Serbie" était avant sa rédemption. La violence sur laquelle cet ordre nouveau et pur est fondé n'est pas distincte de l'ordre lui-même ; ce qui est réel c'est le monde à venir dans lequel le mal n'aura pas sa place et où tout qui est en place sera bien. Cette structure fantasmatique est évidente dans l'histoire que m'a racontée un membre de l'ONU qui se rappelait un échange au cours duquel, après avoir admonesté un milicien serbe ayant pris part à la destruction d'un "beau et ancien vieux quartier" d'une ville bosniaque, celui-ci lui répondit «mais nous construirons un ancien quartier plus beau et nouveau à la place ». 


\section{L'acceptation des discours nationalistes}

22 Dans les pages précédentes j'ai présenté une interprétation de la généalogie de cette logique. Les fantasmes du bien-être à connaître, une fois l'ordre ancien détruit, sont mis en circulation par les démagogues nationalistes. Cependant, une fois que le régime communiste est remplacé par le nouvel ordre nationaliste, la richesse et la prospérité promises ne se concrétisent pas. Ce sont des boucs émissaires déjà tout désignés, des membres d'autres groupes nationaux qui sont perçus à la fois comme une entrave aux intérêts nationaux de l'extérieur et comme des saboteurs de leur réalisation de l'intérieur: ils portent non seulement le blâme pour les iniquités du vieux système, mais aussi la responsabilité des échecs du nouveau. Comme les nouveaux dirigeants nationalistes essaient de gagner des prises plus fermes sur des appareils d'État, ils diabolisent les autres nations en fournissant des "preuves" que ces ennemis sont non seulement opposés au bien-être du peuple, mais qu'ils se consacrent aussi à sa destruction totale. Les stations de radio et les journaux, contrôlés par les gouvernements nationaux, font circuler des histoires suscitant la crainte de meurtres et de mutilations exécutés contre des membres de la communauté nationale par des personnes d'autres nations. En favorisant l'extension de la crainte et de la méfiance, la nouvelle direction légitime son appel à la mobilisation de la nation pour la guerre contre des ennemis extérieurs et intérieurs; de ce fait elle assure sa mainmise sur les appareils d'État répressifs. Miloš Vasić, écrivant à propos de la militarisation des Bosniaques serbes, démontre que «tout d'abord, la propagande chauviniste belliciste est répandue par les médias serbes contrôlés. La peur prend place et l'idée que "nous ne pouvons plus vivre avec eux" devient dominante $»^{29}$. L'acceptation populaire de telles histoires de persécutions engendre elle-même des meurtres et des mutilations dirigées contre l'“autre" qui, en prévention, rend la monnaie de la pièce, donnant ainsi naissance à de nouvelles rumeurs et d'autres histoires d'atrocités commises par l'ennemi. Ainsi que le We Are All Neighbours de Christie et Bringa le montre, l'acceptation de ces rumeurs initie une spirale de méfiance et de violences réciproques détruisant les modèles de sociabilité et les remplaçant par des oppositions basées sur la crainte se manifestant dans les violences pour détruire l'ennemi avant que celui-ci ne puisse détruire l'autre ${ }^{30}$. Les mouvements pour détruire cet ennemi suivent la logique de ce que Riches appelle la "préemption tactique $»^{31}$; tuer des enfants, des femmes et des vieillards afin de les empêcher de devenir, donner naissance, ou aider, ceux qui vont vous tuer a un sens une fois que l'ennemi est reconnu en tant que tel.

Cependant, cette reconnaissance ne peut pas être expliquée uniquement en termes empiriques, surtout lorsque la réalité du conflit contredit les témoignages de la vie quotidienne, comme dans le village décrit dans le documentaire. Il est indubitablement vrai que les forces politiques ont joué un rôle significatif en donnant forme à, et en disséminant, des rumeurs qui ont généré la crainte et donné naissance à la violence inter-communautaire. La raison pour laquelle de telles rumeurs devraient être acceptées comme vraies n'est pas claire, ni non plus, peut-être d'une façon plus frappante, pourquoi elles devraient être perçues comme pertinentes là où aucun signe de haine intercommunautaire n'a été auparavant perçu. L'acuité de la violence se déroulant désormais entre les communautés de l'ancienne Yougoslavie n'était pas manifeste avant la mobilisation nationaliste ; comme Comelia Sorabji le démontre dans l'exemple bosniaque "pour la plupart, la tolérance, la bonne volonté et un désir 
conscient pour des rapports coopératifs et civils assurait une cohésion entre les trois populations ${ }^{32}$. Si nous expliquons la brutalité extrême, si visible aujourd'hui dans l'ancienne Yougoslavie, comme quelque chose d'endémique aux "Balkans", non seulement nous nions de telles vérités ethnographiques et nous ignorons l'histoire récente de la modernisation en Yougoslavie, mais encore nous plaçons effectivement les Yougoslaves au ban de ce que nous appelons la "société humaine" (pour agir ainsi, "ils" doivent être essentiellement différents de "nous"). Si, par contre, nous acceptons que les discours politiques des dirigeants de partis opposés des anciennes républiques aient, d'une manière ou d'une autre, transformé les Yougoslaves en quelque chose de différent de ce qu'ils étaient auparavant, nous sommes encore face à la question de la provenance de ce penchant pour la violence extrême. Peter Loizos, confronté à des exemples analogues de violence génocidaire dans le contexte chypriote ${ }^{33}$, soutenait que la violence ethnique était concentrée sur un ensemble spécifique de sujets par des rhétoriques politiques antagonistes. Cependant, il laissait de côté le problème de savoir comment ces rhétoriques pouvaient si bien atteindre leur cible et pousser des individus à associer l'image de l'autre à celle de l'ennemi avec tant de passion que la volonté d'effacer sa présence de la terre dépasse les scrupules moraux ayant régulé l'interaction sociale avant que l'autre ne soit considéré comme tel.

Tandis que l'argument de la "mentalité balkanique" manifeste une indolence intellectuelle dans la mesure où il mobilise des stéréotypes de sens commun et racistes afin d'ignorer le défi de la compréhension d'autres cultures, celui de la rhétorique politique, quant à lui, ignore le défi qu'offrent les situations concernant les communautés qui, ayant vécu ensemble, en paix et en coopération, se fragmentent soudainement en factions guerrières aux conceptions modernistes de la nature humaine. Si, comme les théories des Lumières sur la nature humaine le prétendent, l'être humain agit rationnellement et coopérativement lorsqu'il a le choix, alors il n'y a aucune raison, quand les options présentées sont soit un modèle prouvé de cohabitation, soit un paradigme radical d'affrontement violent, pour que le choix doive être fait en faveur de l'antagonisme intercommunautaire et de la guerre. Bien que j'aie montré dans les pages précédentes que la dernière option était offerte aux peuples yougoslaves par des factions politiques opportunistes, je n'ai pas été capable de démontrer une raison "rationnelle" de l'acception par les citoyens de la logique de la haine intercommunautaire comme plus vraisemblable que leurs propres expériences de cohabitation et de coopération. $\mathrm{Si}$, comme Mastnak l'affirme, la situation actuelle est une expression de la volonté populaire, alors il est important d'essayer de discerner ce qui, dans la population, répond, avec une rage apparemment primitive, à l'appel à la destruction d'un ennemi avant que celui-ci n'en soit lui-même capable. Je suggère, à la suite de Jacques Lacan, que nous devons regarder au-delà de la rhétorique des discours sociaux, vers ces fantasmes primitifs mobilisés par ces rhétoriques. Ces fantasmes, générés par la première rencontre du nourrisson avec l'ordre symbolique, répondent, et obligent le sujet à répondre, à l'appel qui commande d'infliger une violence absolue à un ennemi absolu.

L'entrée de l'enfant dans l'ordre symbolique, lorsqu'il apprend qu'il doit faire appel à quelqu'un d'autre pour réaliser ses désirs, est simultanément l'expulsion d'un monde dans lequel il "se souvient", ensuite, avoir eu tout ce qu'il voulait. Freud, dans l'introduction de Malaise dans la civilisation, énonce que «le nourrisson ne différencie pas encore son moi d'un monde extérieur comme la source des sensations qu'il reçoit » et que cette expérience peut donner naissance à des souvenirs incomplets de 
《sentiment océanique », tel un «narcissisme illimité ${ }^{34}$. Dans cet état pré-linguistique, l'enfant n'a aucun appareil conceptuel lui permettant de distinguer l' "intérieur" de l'“extérieur", et il se perçoit ainsi lui-même comme locus et source de sensation, ce qui donne naissance à la sensation. L'apprentissage par l'enfant de la langue supprime ce sens d'omnipotence narcissique en réorganisant le monde en terme de dualisme; en se séparant de la mère, l'enfant qui appréhendait le monde et lui-même comme un tout indifférencié prend conscience que, non seulement, il est une partie du monde, mais, qu'en outre, il en est une petite partie sans défense devant faire appel à d'autres ayant le pouvoir de lui donner, et de le priver, de ce qu'il désire. Après l'appréhension du monde par la langue, le "plaisir" primitif (que Lacan appelle jouissance) ne demeure que comme la trace d'une absence (Lacan écrit « nous devons souligner que la jouissance est interdite à celui qui en parle $\left.»^{35}\right)$. Cette absence, ou ce manque, sert d'écran pour projeter nos fantasmes d'accomplissement, de plaisir total, sous la forme d'objets ou de scénarios de désir. Ces "objets intégrants", qui, en tant que fétiches, remplacent la jouissance qui a été irrémédiablement perdue, semblent promettre l'accès à la réalisation de ce que la langue nous a interdit. En ce sens, ils recouvrent l'abîme de ce manque primitif et nous permettent de fantasmer que « si nous avions cette chose nous aurions notre bonheur (jouissance) ». Ainsi, bien que ce manque ne puisse jamais être rien de plus de ce côté-ci du langage que la blessure d'une perte, il demeure néanmoins le domaine dans lequel nous inscrivons les désirs qui animent nos agissements sublimés. L'idée de perte, de quelque chose de brutal qui a été fait afin de nous couper de cette partie de nous-mêmes qui nous donnait notre plaisir, conduit, bien sûr, à la question «qui nous a fait cela?». Dans les termes de Lacan, ce violeur est celui qui nous fait connaître les fondements de la langue en nous introduisant, en tant que nourrissons, à la présence et à l'absence (le soi et le non-soi), quand il a demandé que la mère laisse l'enfant et vienne à lui. Bien que Freud appelle ce personnage «le Père ", il ne requiert ni d'être personnifié ni d'être engendré, c'est quelque chose/quelqu'un d'extérieur à l'union du corps de l'enfant avec ce qui le nourrit, le console et le soutient, que l'enfant, dans son incursion initiale dans la signification, reconnaît comme brisant cette union par l'assertion de sa présence, sa « voix ».

Cependant, une fois que l'enfant vient à reconnaître la nécessité d'opérer dans l'ordre symbolique, il canalise ses désirs sur certains modèles de comportement par l'apprentissage que certaines activités fourniront l'accomplissement (et d'autres la punition). Par son expérience de la récompense et de la privation parentales, il en arrive à constituer pour lui-même une image («l'idéal du moi ») de ce qu'il doit être pour gagner l'amour de ceux qu'il désire et les choses avec lesquelles ces autres peuvent le fournir. Cette image du "bon soi" sert, par l'intériorisation de ce que l'enfant perçoit comme étant ce que les parent veulent qu'il soit, à établir l'identité de l'enfant dans des modèles normatifs de motivation et d'attente. Néanmoins, ce procédé apparemment rationnel d'acculturation fonctionne par un procédé de déplacement temporaire par lequel l'enfant imagine qu'il sera encore capable de réaliser tous ses désirs bien qu'il doive modifier ses tactiques pour correspondre aux demandes de ses parents. La volonté narcissique du pouvoir souligne encore la relation de l'enfant avec l'ordre symbolique. C'est seulement en négociant le complexe d'ÆEdipe que l'enfant apprend qu'il y a des limites à son désir qui ne peuvent pas être franchies. Le complexe d'Edipe est résolu lorsque l'enfant, qui, jusqu'à ce temps, continue d'exiger le corps de la mère (le premier substitut de fétiche pour la jouissance) comme l'objet de son désir, est "convaincu" qu'il doit, dans son propre intérêt, abandonner cette exigence. Cela 
survient, dans des manières qui diffèrent selon le sexe de l'enfant, lorsqu'il est amené à réaliser que, s'il continue à exiger ce que ni la société ni la voix parentale qui "parle" pour la société ne lui accordera, il sera privé de la possibilité de tout plaisir futur par ce que l'enfant reconnaît, selon Freud, comme une "castration" ${ }^{36}$. La menace de castration est, par conséquent, intériorisée dans le "sur-moi", qui sert effectivement à rappeler à l'enfant, et à l'adulte qu'il devient, que s'il doit jamais éprouver quelque plaisir que ce soit, certains objets de désir doivent être abandonnés et remplacés par des objets que la société reconnaît comme appropriés. Autrement dit, la personne correctement socialisée est celle qui reconnaît que le plein assouvissement, le retour à la jouissance que le fantasme d'œdipe évoque avant que la menace de castration ne le ramène dans l'inconscient, est rendu impossible par la "réalité".

Néanmoins, des traces de cette construction difficile de l'identité individuelle demeurent inscrites dans l'inconscient. Les individus rencontreront toujours, au travers de toutes leurs activités, des échecs dans leurs stratégies d'accomplissement. De tels moments évoquent fréquemment le scénario prélinguistique dans lequel un ennemi général est placé en opposition à un fantasme d'accomplissement et de plaisir. Dans de tels cas, l'échec à réaliser l'accomplissement est éprouvé comme une conséquence des activités de l'ennemi "démoniaque", que l'enfant a déjà rencontré lorsque son omnipotence primitive fut brisée par la "voix du Père". Quand la frustration du désir évoque la présence fantasmatique de cet adversaire, perçu dans les termes infantiles comme un être qui existe seulement pour voler tout ce que possède l'enfant afin de se faire plaisir, les individus peuvent répondre en dirigeant la rage et la violence primitives contre ce qu'ils perçoivent comme la source de cette frustration. Dans la plupart des cas, cependant, de telles éruptions de matières inconscientes à la vie consciente sont postérieurement interprétées (par l'acteur et le récepteur de sa violence) comme un comportement irrationnel (c'est-à-dire une "crise douce") et elles sont refoulées dans l'inconscient par le sur-moi de la personne. Cependant, certains individus, ayant échoué à intérioriser les exigences de "réalité" dictées par le sur-moi, imposent la logique d'une structure psychique, polarisée entre le désir et la haine, au domaine de leurs relations avec la société. Ainsi, ils interprètent le monde en termes de dualisme divisant tous les éléments du domaine social en ami et ennemi (le soi et l'Autre). Dans la plupart des cas, de telles personnes sont perçues comme paranoïaques et, si leur violence se révèle de manière endémique pertubatrice, elles sont institutionnalisées. Cependant, certaines structures discursives utilisent l'opposition psychique d'ennemi et de moi en établissant comme réel et normatif un monde polarisé entre des ennemis inflexibles et une communauté menacée par eux ${ }^{37}$. Cette structure inconsciente a servi aux formes du nationalisme mobilisées en Serbie et en Croatie (mais qui étaient absentes en Slovénie en raison des difficultés à invoquer de manière convaincante la présence d'un ennemi démoniaque) et elles ont mobilisé les passions y étant liées en définissant la "vraie" nation comme l'objet qui recouvre ce manque. Dans ces rhétoriques nationalistes, tout accomplissement réel découle de la réalisation de la Nation, et l'“autre" (qu'il soit juif, Croate, Musulman, Serbe, Albanais, etc.) est inscrit dans cette rhétorique précisément comme ce qui a pour seule raison d'être le désir de nier, voler et détruire l'identité nationale qui fournit à quiconque ce qu'il veut et fait de lui ce qu'il est réellement. L'"autre" vole la terre, viole des femmes, désacralise les objets sacrés et, enfin, anéantit la communauté dans laquelle celui-là trouve une identité. Ces rhétoriques non seulement définissent la Nation comme la "Chose" qui 
récupère la jouissance, mais encore érigent la Nation des "autres" en incarnation de l'ennemi démoniaque menaçant le plaisir à la racine même de son être ${ }^{38}$.

\section{La dynamique nationaliste}

Cependant, il est important de reconnaître que l'identification des individus avec la structure établie dans le discours nationaliste est dynamique, et c'est le caractère de processus de cette interpellation qui permet à la rhétorique nationaliste d'évoquer les structures psychiques inconscientes. La libération de ce qu'elle définit comme répression ennemie et la légitimation des désirs qu'elle énonce comme essentiels et réalisables fournissent les fondations des projets pour les sujets de l'appel nationaliste promettant non seulement de restaurer la vraie nation, mais encore de réaliser leurs identités authentiques pour eux. Durant la période de l'hégémonie communiste (une hégémonie établie par Tito et célébrée, jusqu'à la chute du communisme, sous le regard omniprésent de ses portraits), les "plaisirs" de l'identification nationale étaient explicitement proscrits par l'idéologie de bratstvo $i$ jedinstvo; on affirmait aux Yougoslaves, et ils en étaient convaincus, qu'ils devaient abandonner le fantasme de l'identité nationale afin de garantir la survie et la construction d'un système social qui pourrait leur fournir le bien-être. Autrement dit, l'idéologie socialiste servit de forme de sur-moi social dans la mesure où elle affirmait que si les individus devaient continuer à demander la réalisation de leurs aspirations nationalistes, ils seraient détruits par les activités d'ennemis extérieurs. L'effondrement de l'idéologie communiste survint lorsque l'identité supranationale promulguée par l'État yougoslave vint à être interprétée, non pas comme quelque chose fonctionnant pour le propre intérêt des Yougoslaves, mais comme quelque chose leur étant imposé par des "ennemis extérieurs" (le "croate" Tito ou les "hégémonistes serbes"). La proscription par l'État yougoslave du nationalisme vint à être perçue, non pas comme une règle générale qu'on devait suivre pour survivre et prospérer dans le monde réel, mais comme une manifestation d'antagonisme, et, par conséquent, Tito et l'ordre qu'il représentait devinrent des "ennemis du peuple". En outre, le fantasme nationaliste devint, non pas quelque chose d'impossible et d'autodestructeur, mais quelque chose qui pourrait, et devait, être réalisé. Le domaine discursif fut transformé en ce qu'Adorno et Horkheimer appellent une structure "paranoïde" 39 par la popularisation de la croyance que la possession du "réel" objet de désir du peuple (la nation) était possible et empêchée seulement par la présence d'autres dont l'unique raison d'être était de nier cet objet aux individus.

Cette structure fut mise en place par la propagande évoquant, simultanément, d'une part, le futur "restauré" de la nation comme un objet fantasmatique promettant la récupération utopique des plaisirs perdus lorsque, dans un passé brumeux, les individus furent exilés de leur "patrie", et, d'autre part, un ennemi démoniaque se dressant comme l'antithèse physique de toutes les configurations de volonté et de désir. Cependant, tandis que la "terre natale" promise est esquissée dans ces rhétoriques nationalistes en termes édéniques encore imprécis, le mal de l'ennemi et la dévotion héroïque du dirigeant national à son extirpation sont dépeints avec un réalisme graphique. Dans le fantasme nationaliste, c'est le dirigeant et l'ennemi qui sont les éléments cruciaux, et opératoires. On se bat contre l'ennemi sous la direction du dirigeant afin de "recouvrer" la nation, mais, puisque l'accès au pur plaisir d'être, 
que les rhétoriques nationalistes revendiquent, sera fourni par la défaite de l'ennemi et reste toujours bloqué par les limitations des réalités sociales et psychiques, la destruction de l'ennemi se montrera toujours inadéquate. Ce qui est implicite dans la structure psychique sur laquelle la rhétorique nationaliste se dessine, c'est la spirale de violence conduisant les membres de la communauté nationale à toujours rechercher, au moment de la victoire, encore un autre ennemi qui peut être blâmé pour le fait que la nation "réelle" ne soit pas à l'endroit qu'ils ont juste récupéré à l'ennemi défait ${ }^{40}$. Si les Nazis avaient eu l'occasion d'exterminer chaque gauchiste, juif, estropié, homosexuel, et gitan qui pouvait être blâmé pour empêcher l'avènement du "Reich millénaire", qu'ils voyaient comme leur héritage réel, ils auraient eu à commencer par exterminer ces Allemands qui, bien que remplissant tous les critères de "purs Allemands", étaient néanmoins les causes de l'échec de la matérialisation du Millénaire. Vesna Pešić suggère que la même logique opère dans l'exemple serbe, lorsqu'elle écrit qu'« après le nettoyage ethnique, nous aurons bientôt le nettoyage des traîtres $»^{41}$.

\section{Conclusion}

Je soutiens que les rhétoriques nationalistes ayant conduit à la guerre dans l'ancienne Yougoslavie fonctionnent en incitant des individus aux bagages sociaux et historiques très divers à reconnaître leurs identités essentielles comme nationales plutôt que comme basées sur le sexe, la profession, la classe, ou le lieu de résidence. Elles y parviennent par la construction discursive d'ennemis de la nation qui, non seulement servent de boucs émissaires responsables de tout qui va de travers, aussi bien dans la société que dans les vies de ses membres, mais servent encore à évoquer, par leur négativité, une positivité nationale, sur laquelle les individus peuvent fantasmer et qui émergera soudainement et paradisiaquement si l'ennemi est détruit. J'ai suggéré, à la suite de Freud et de Lacan, que ce processus de création d'une ferveur nationaliste réussit parce qu'il fait écho, dans la sphère sociale, aux processus de formation de l'identité que les individus négocient dans leurs premières rencontres avec la réalité sociale. La violence de l'entrée du nourrisson dans l'ordre symbolique se reflète dans les scénarios violents par lesquels la propagande nationaliste présente l'ennemi de la nation aux modes de vie différents de la communauté nationale, et je soutiens qu'elle représente le lien entre ces deux "scènes" qui oblige des individus, indépendamment de leurs expériences d'adulte, à se sentir concernés par l'appel à joindre la lutte nationale.

Les interprétations psychanalytiques de l'action sociale sont perçues par la plupart des anthropologues sociaux comme profondément contraires au mode de vie de la communauté académique à laquelle ils doivent allégeance. C'est parce qu'il semble que la psychanalyse défie la supposition axiomatique sur laquelle elle est fondée : la vérité $a$ priori que la réalité sociale est une construction sociale. La remarque que j'ai tenté de faire ici ne s'oppose cependant pas à cet axiome. Elle suggère plutôt que, dans la mesure où les hommes agissent dans la société parce qu'ils reconnaissent les identités qu'elle leur procure comme étant les leurs, nous devons alors chercher à comprendre les processus par lesquels des individus "se reconnaissent" eux-mêmes dans les positions de sujet fournies par des discours sociaux. Je prétends que le domaine de 1'“irrationnel", que dénient les discours analytiques basés sur les suppositions de Descartes au sujet de rationalité et d'identité, est ce qui oblige des personnes à désirer adopter, et défendre, les identités culturelles offertes dans les discours sociaux. 
L'exemple de l'ancienne Yougoslavie est, d'une certaine façon, un cas extrême, mais, ainsi que je l'ai soutenu, il y a aussi bien une logique sociale opérant dans ces discours politiques qui font d'anciens voisins des ennemis de sang, qu'une logique d'identification, qui souligne des moments inscrits dans l'inconscient humain par la première rencontre $d u$ nourrisson avec l'ordre social, lequel oblige les anciens Yougoslaves à adopter passionnément les identités nationales belliqueuses professées par ces discours politiques. J'estime que ni cette logique sociale, ni les structures de l'inconscient qu'elle mobilise ne pourraient créer indépendamment les sociétés inciviques que nous voyons actives dans l'ancienne Yougoslavie; mais réunies, elles engendrent des réalités sociales logiques, auto-affirmées, capables de se soutenir et de se reproduire. D'autres articulations du social et de l'inconscient créent d'autres ordres sociaux, moins "extrêmes", où les oppositions sont diversement réparties sur les nombreuses rencontres sociales que les individus font dans leurs vies quotidiennes. L'ancienne Yougoslavie est un exemple extrême seulement dans la mesure où ses politiciens ont si efficacement réussi à transposer les fantasmes du nationalisme aux structures inconscientes d'antagonisme. Cependant, une telle juxtaposition n'est pas anormale, et d'autres nationalismes actifs aujourd'hui en Europe de l'est, et au-delà, engagent la construction analogue d'identités essentielles idéalisées et d'identités démoniaques. L'appel de telles articulations discursives est profond, et fournit aux nationalistes, se reconnaissant dans les identités professées, aussi bien des modèles d'interprétation puissants et logiques que des motifs d'action. Pour comprendre de tels individus, et les communautés qu'ils forment, nous devons, en tant qu'anthropologues, prêter attention tant aux discours par lesquels leur réel est constitué qu'aux processus d'identification par lesquels ils appréhendent ces réalités sociales comme lieux de reconnaissance et d'action.

\section{NOTES}

1. Hobsbawm (Eric), Nations and Nationalism since 1780: Programme, Myth, Reality, Cambridge: Cambridge University Press, 1990, pp. 182-183.

2. Gaber (S.), " The limits of democracy : the case of Slovenia ", Journal of Area Studies, (3), 1993.

3. Cité dans Thompson (Mark), A Paper House: the Ending of Yugoslavia, London: Hutchinson Radius, 1992, p. 104.

4. Voir le documentaire du Disappearing World «We Are All Neighbours", dirigé par Debbie Christie et basé sur le travail de Tone Bringa

5. Althusser (Louis), «Ideology and ideological state apparatuses (notes towards an investigation) ", in Lenin and Philosophy and Other Essays, London : Verso, 1971, pp. 162-163.

6. Mastnak (Tomaz), « Civil society at war », Yugofax, (16), 1992, p. 7.

7. Voir Hammel (E. A.), «Demography and the origins of the Yugoslav civil war ", Anthropology Today, 9 (1), 1993.

8. van den Heuvel (M.), Siccama (J. G.), eds., The Disintegration of Yugoslavia, Amsterdam : Rodopi, 1992, frontispice. 
9. Cf. Dimitrijevic (V.), «Ethnonationalism and the constitutions : the apotheosis of the nation state ", Journal of Area Studies, (3), 1993 et Hayden (Robert), "Constitutional nationalism in the formerly Yugoslav republics ", in Bowman (Glenn), ed., Antagonism and Identity in the National Idiom : the Case of Former Yugoslavia, Oxford : Berg Press, 1994.

10. Denich (Bogdan), «Unbury the victims: rival exhumations and nationalist revivals in Yugoslavia ", American Anthropological Association Annual Meeting, Chicago, 1991, p. 2.

11. Auty (Phillis), «The post-war period", in Clissold (Stephen), ed., Short Historv of Yugoslavia from Early Times to 1966, Cambridge : Cambridge University Press, 1966, p. 247.

12. Cf. Hobsbawm (Eric), "Introduction », in Hobsbawm (E.), Ranger (T.), eds., The Invention of Tradition, Cambridge : Cambridge University Press, 1983.

13. Cf. Pavlowitch (Stevan K.), The Improbable Survivor: Yugoslavia and its Problems 1918-1988, London : C. Hurst and Co, 1988, pp. 105-106.

14. Allcock (John B.), «Rhetorics of nationalism in Yugoslav politics », in Allcock (John B.), Hoarton (John J.), Milivojevic (Marko), eds., Yugoslavia in transition. Choices and Constaints, New York / Oxford : Berg, 1992, p. 283.

15. Ardener (E.), «Introductory essay : social anthropology and language », in Ardener (E.), ed., Social Anthropology and Language : ASA Monograph X, London : Tavistock, 1971, p. xliv.

16. Allcock (John B.), art.cit., pp. 281-287.

17. Cf. Plestina (D.), «Economic development and nationalism: the Yugoslav lesson», in Bowman (Glenn), ed., op.cit.

18. Ramet (Sabrina P.), Nationalism and Federalism in Yuroslavia: 1962-1991, Bloomington: Indiana University Press, 1992 (Second Edition), p. 200.

19. Dukic (D.), « An overview of important events in Croatian history », in Feldman (L.), Prica (I.), Senjkovic (R.), eds., Fear, Death and Resistance: An Ethnography of War. 1991-1992, Zagreb : Matrix Croatica, 1993, p. 251.

20. Tudjman (Franjo), Bespuca povijesne zbilnosti, Zagreb : Matrix Croatica, 1990.

21. Salecl (R.), « Nationalism, anti-semitism and anti-feminism in Eastern Europe », Journal of Area Studies, (3), 1993.

22. Denich (Bogdan), art.cit., p. 6.

23. Salecl (R.), art.cit., p. 81 ; Bloch (M.), « Death, women and power », in Bloch (M.), Parry (J.), eds., Death and the Regeneration of Life, Cambridge : Cambridge University Press, 1982 ; Bloch (M.), "Almost eating the ancestors", in Ritual. History and Power : Selected Papers in Anthropology, London: Athlone Press, 1989, p. 170.

24. Denich (Bogdan), art.cit., p. 11.

25. Voir les pamphlets du Ministère de l'information de Bulajic (M.), Never Again: Genocide of the Serbs, Jews and Gypsies in the Ustashi Independent State of Croatia, Belgrade : Ministry of Information of the Republic of Serbia, 1991 et Kljakic (S.), A Conspiracy of Silence: Genocide in the Independent State of Croatia and Concentration Camp Jasenovac, Belgrade : Ministry of Information of the Republic of Serbia, 1991.

26. Cf. Bowman (Glenn), «Nationalizing the sacred: shrines and shifting identities in the Israeli-occupied territories ", Man: The Journal of the Royal Anthropological Institute, 28 (3), 1993.

27. Kapferer (B.), Legends of People/Myths of State: Violence, Intolerance and Political Culture in Sri Lanka and Australia, Washington, D.C. : Smithsonian Institution Press, 1988.

28. Cf. Bax (M.), «Marian apparitions in Medjugorje; rivalling religious regimes and state formation in Yugoslavia ", in Wolf (Eric), ed., Religious Regimes and State-Formation: Perspectives from European Ethnology, Albany : State University of New York Press, 1991 et Bax (M.), « How the mountain became sacred : antagonism and the construction of religious community in a Yugoslav rural parish ", in Bowman (Glenn), ed., op.cit. sur le développement de ce sanctuaire.

29. Vasic (M.), "The pattern of aggression : two against one in Bosnia ", Balkan War Report, 18, 1993, p. 8. 
30. Cf. Riches (D.), "The Phenomenon of Violence», in Riches (D.), ed., The Anthropology of Violence, Oxford : Basil Blackwell, 1986 ; Loizos (P.), «Intercommunal killing in Cyprus », Man : The Journal of the Royal Anthropological Institute, 23 (4), 1988.

31. Riches (D.), art.cit., pp. 6-7.

32. Sorabji (C.), «Ethnic war in Bosnia?», Radical Philosophy, (63), 1993, pp. 33-34. Cf. aussi Bringa (Tone), "We are the way our surroundings are" : identity formation in an ethnically mixed village in Bosnia », in Bowman (Glenn), ed., op.cit.

33. Loizos (P.), art.cit., p. 651.

34. Freud (Sigmund), Civilization and its Discontents, London : The Hogarth Press, 1963, pp. 3-4, 9.

35. Lacan (Jacques), Écrits : A Selection, London : Tavistock Publications, 1977, p. 319.

36. Mitchell (J.), Psychoanalysis and Feminism, Harmondsworth : Penguin Books, 1974, pp. 74-100.

37. Adorno (T.), Horkheimer (M.), Dialectic of Enlightenment, London : Verso, 1972, p. 187.

38. Cf. Zizek (S.), « Eastern Europe's Republics of Gilead », New Lef Review, (183), 1990.

39. Adorno (T.), Horkheimer (M.), op.cit., pp. 179-200.

40. Zizek (S.), Looking Away: An Introduction to Jacques Lacan through Popular Culture, Cambridge, Mass. : M.I.T. Press, 1991, p. 6.

41. Pesic (Vesna), « The problems of minority rights in new nation states », Yugofax, (1005), 1992, p. 7.

\section{RÉSUMÉS}

Le rationalisme moderniste ne nous aide pas dans l'analyse du nationalisme ou de la haine nationale. A la suite des élections yougoslaves de 1990 sont arrivés au pouvoir des partis politiques prétendant représenter le peuple, le groupe national dominant dans chaque république et affirmant que leur développement était freiné par les autres. Si la haine nationale a été provoquée d'en haut, la réponse populaire a tout de même été enthousiaste. Après avoir analysé les moyens de manipulation, l'auteur s'intéresse aux raisons de son acceptation. L'appel à la haine de l'autre réussit à s'ancrer dans des individus aux bagages culturels et sociaux différent parce qu'il fait écho aux processus de formation de l'identité.

The modernist rationalism is not that helpful in the analysis of nationalism or national hatred. The polical parties that took power in the 1990s, have claimed that they represented the people, the dominant ethnic groupings of the respective republics and have asserted that their development was impeded by the others. If ethnic hatred has been instigated from above, the people's response, yet, was enthusiastic. Firstly, the author analyses the means of manipulation. Secondly, he looks for the reasons of its acceptation. The call of other's hatred was successful among persons of a widely range of social and historical backgrounds because it echoes processes of identity formation.

\section{INDEX}

Mots-clés : Bratsvo i jedinstvo, Nation, Nationalisme 


\section{AUTEURS}

\section{GLENN BOWMAN}

G. Bowman est le directeur de Communications \& Image Studies, et professeur d'anthropologie à l'University of Kent. 